\title{
The Notions of Horizon and Expectancy in Dynamic Economics
}

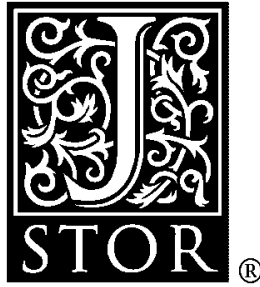

\section{J. Tinbergen}

Econometrica, Vol. 1, No. 3. (Jul., 1933), pp. 247-264.

Stable URL:

http://links.jstor.org/sici?sici=0012-9682\%28193307\%291\%3A3\%3C247\%3ATNOHAE\%3E2.0.CO\%3B2-0

Econometrica is currently published by The Econometric Society.

Your use of the JSTOR archive indicates your acceptance of JSTOR's Terms and Conditions of Use, available at

http://www.jstor.org/about/terms.html. JSTOR's Terms and Conditions of Use provides, in part, that unless you have obtained prior permission, you may not download an entire issue of a journal or multiple copies of articles, and you may use content in the JSTOR archive only for your personal, non-commercial use.

Please contact the publisher regarding any further use of this work. Publisher contact information may be obtained at http://www.jstor.org/journals/econosoc.html.

Each copy of any part of a JSTOR transmission must contain the same copyright notice that appears on the screen or printed page of such transmission.

JSTOR is an independent not-for-profit organization dedicated to and preserving a digital archive of scholarly journals. For more information regarding JSTOR, please contact support@jstor.org. 


\section{THE NOTIONS OF HORIZON AND EXPECTANCY IN DYNAMIC ECONOMICS ${ }^{1}$}

\section{By J. Tinbergen}

IN A THEORY of economic dynamics, the ophelimity function of individuals must be supposed to depend on the quantities of goods consumed and the sacrifices brought, not only at the moment considered, but also at later moments. Their offer and demand schemes for each moment then depend not only on the prices governing at that moment, but also on the price expectances the individuals have for the future. Among those expectances, those relating to the near future will be of more importance than those relating to a further period. As a first approximation it might be supposed that only the expectances relating to a certain time period (the "horizon") are of importance, and all of the same importance. That means that the subject is at every moment $t$ making a definite plan for the period from $t$ to $t+\tau$, and then realizes certain parts of that plan. Before other parts could be realized, the subject makes a "revision" at the moment $t+1$, say, for the period from $t+1$ to $t+\tau+1$, etc.

The purpose of the present paper is to discuss, with the help of these notions, some results of statistical analysis, which cannot be explained by static theory, and which seem to teach something about horizon or expectances.

\section{RELATION BETWEEN TOTAL ANNUAL SUPPLY OF NON-PERISHABLE CROPS AND AMOUNTS HANDLED}

The amount handled is always smaller than the total supply (crop plus carry-over), the difference being the next year's carry-over. The static theory does not yield us a principle to calculate the proportion of sellings to total supply. I suggested a very much simplified scheme -in which the above-mentioned notions appear-to solve the problem, in Zeitschrift für Nationalökonomie, III (1931), p. 169.

In this paper I assumed that

1. demand has the form

$$
v_{t}=f\left(p_{t}\right)+\alpha t
$$

$p_{t}$ being the average price during year $t, \alpha$ a constant;

2 . cost of carrying over may be neglected;

${ }^{1}$ I am indebted to Prof. Th. Limperg, of the University of Amsterdam, for several critical remarks, and to the Netherlands' Central Bureau of Statistics for lending the clichés. 
3. expectances of further crops and demand may be "reasonable," which means that (A) crop expectances are supposed to be equal to the average crop falling on the acreage and to increase every year with $\alpha$; (B) demand expectance is supposed to be in the same relation with price expectance as actual demand with actual price.

I reached the conclusion that ${ }^{2}$

$$
v_{t}=\bar{e}_{t}+\frac{1}{\tau}\left(E_{t}-\bar{e}_{t}\right)
$$

$\bar{e}_{t}$ being the normal (average) drop for each year; $E_{t}$ being the actual supply at beginning of crop year; $\tau$ being an average horizon of sellers.

This result may be generalized by assuming that apart from the carry-over considered in the above formula and the paper mentioned, there exists a certain stock to meet with the risks of unexpected changes; this supply may be supposed to be proportional to $\bar{e}$,and so the total real supply measured will be

$$
s_{t}=E_{t}+\beta \bar{e}_{t} .
$$

Formula (1) now turns into:

$$
v_{t}=\bar{e}_{t}+\frac{1}{\tau}\left[s_{t}-\beta \bar{e}_{t}-\bar{e}_{t}\right]=\gamma \bar{e}_{t}+\frac{1}{\tau} s_{t} .
$$

It is possible to test statistically this relation and to determine $\boldsymbol{\tau}$. as soon as a series of annual figures on $v_{t}$ and $s_{t}$ are available- $\bar{e}_{t}$ assumed to be equal to the trend value of $v_{t}$. Such data are indeed available for several important crops; those for coffee, cotton, wheat, and sugar, are given in Tables I and II. From the theoretical deduction it is clear that as supply we shall have to consider the sum of crop and carry-over as far as the latter is in the possession of producers and of dealers. Stocks held by consumers should not be taken account of. It is, however, difficult to tell, and perhaps interesting to discuss at a later opportunity, whether, for instance, importers' stocks should be included or not. When the situation is such that the great bulk of stocks is held by importers, as is the case for coffee before the war, it is clear that those stocks should be included; for our deduction relates especially to those economic subjects that bear the risk of carrying over. The figures for coffee given in Table I therefore include those stocks. The figures for wheat and cotton do not include them; for

2 As other results of this analysis, one finds two relations that are in good accordance with facts, viz., (1) equality of prices and price expectances for next year except in case of a very short crop; (2) inverse correlation between average price during crop year and carry-over at end of crop year. 
TABLE I

Total Supply and Deliveries of Coffee, 1884-1913 and 1920-1931 (Millions of Sacks)

\begin{tabular}{|c|c|c|c|c|c|}
\hline Year & $\begin{array}{c}\quad v_{t} \\
\text { a. World } \\
\text { Deliveries }\end{array}$ & $\begin{array}{l}s_{t} \\
\text { b. Total } \\
\text { Supply }\end{array}$ & $\begin{array}{c}e_{t} \\
\text { c. Trend } \\
\text { of Deliveries }\end{array}$ & $\begin{array}{c}\mathrm{C} \text { in } \% \\
\text { of } \mathrm{c}\end{array}$ & $\begin{array}{l}\mathrm{a} \text { in } \% \\
\text { of } \mathrm{c}\end{array}$ \\
\hline 1884 & 9.4 & 14.8 & 8.7 & 170 & 108 \\
\hline 1885 & 10.6 & 15.7 & 9.0 & 174 & 118 \\
\hline 1886 & 10.1 & 14.1 & 9.3 & 152 & 109 \\
\hline 1887 & 10.0 & 14.3 & 9.6 & 149 & 104 \\
\hline 1888 & 8.1 & 11.4 & 9.9 & 115 & 82 \\
\hline 1889 & 9.2 & 12.1 & 10.2 & 119 & 90 \\
\hline 1890 & 9.4 & 12.2 & 10.5 & 116 & 89 \\
\hline 1891 & 8.7 & 11.1 & 10.8 & 103 & 81 \\
\hline 1892 & 10.8 & 13.5 & 11.1 & 122 & 97 \\
\hline 1893 & 10.9 & 14.0 & 11.4 & 125 & 96 \\
\hline 1894 & 10.6 & 13.0 & 11.7 & 111 & 91 \\
\hline 1895 & 11.2 & 13.9 & 12.0 & 116 & 93 \\
\hline 1896 & 11.1 & 14.7 & 12.3 & 120 & 90 \\
\hline 1897 & 12.2 & 16.2 & 12.6 & 129 & 97 \\
\hline 1898 & 14.6 & 21.0 & 12.9 & 165 & 113 \\
\hline 1899 & 13.5 & 20.1 & 13.2 & 152 & 102 \\
\hline 1900 & 14.0 & 21.6 & 13.5 & 160 & 104 \\
\hline 1901 & 14.3 & 25.2 & 13.8 & 183 & 104 \\
\hline 1902 & 15.9 & 28.7 & 14.1 & 204 & 110 \\
\hline 1903 & 16.0 & 29.8 & 14.4 & 207 & 111 \\
\hline 1904 & 16.1 & 29.7 & 14.7 & 202 & 109 \\
\hline 1905 & 16.2 & 28.8 & 15.0 & 192 & 108 \\
\hline 1906 & 16.7 & 30.5 & 15.3 & 200 & 109 \\
\hline 1907 & 17.5 & 34.2 & 15.6 & 219 & 112 \\
\hline 1908 & 17.5 & 33.3 & 15.9 & 210 & 110 \\
\hline 1909 & 18.6 & 35.2 & 16.2 & 217 & 115 \\
\hline 1910 & 18.2 & 32.4 & 16.5 & 197 & 110 \\
\hline 1911 & 17.2 & 30.8 & 16.8 & 184 & 102 \\
\hline 1912 & 17.5 & 30.9 & 17.1 & 180 & 102 \\
\hline 1913 & 17.1 & 30.8 & 17.4 & 177 & 98 \\
\hline 1920 & 14.4 & 23.1 & 17.5 & 132 & 82 \\
\hline 1921 & 18.1 & 27.5 & 18.0 & 153 & 100 \\
\hline 1922 & 17.9 & 25.9 & 18.5 & 140 & 97 \\
\hline 1923 & 19.8 & 24.3 & 19.0 & 128 & 104 \\
\hline 1924 & 20.7 & 30.7 & 19.5 & 158 & 106 \\
\hline 1925 & 19.0 & 28.5 & 20.0 & 142 & 95 \\
\hline 1926 & 20.6 & 31.5 & 20.5 & 154 & 100 \\
\hline 1927 & 21.6 & 39.9 & 21.0 & 190 & 103 \\
\hline 1928 & 21.8 & 40.5 & 21.5 & 188 & 102 \\
\hline 1929 & 21.7 & 46.7 & 22.0 & 212 & 99 \\
\hline 1930 & 22.6 & 51.9 & 22.5 & 230 & 100 \\
\hline 1931 & 23.8 & 58.2 & 23.0 & 254 & 104 \\
\hline
\end{tabular}

Sources and Method of Calculation: World Deliveries from: Roth, Die Übererzeugung der Welthandelsware Kaffee, 1790-1929. Total Supply calculated by adding Stock at end of year to World Deliveries during year. Trend of Deliveries: free-hand straight line trend. Even rather important variations in constants of Trend do not affect materially the result for our purposes. 
wheat this makes only a small difference in the result for $\tau$; for cotton, however, the difference is of more importance; and $\tau$ is larger when importers' stocks are included.

TABLE II

Sopply and Disappearance, Wheat, American Cotton, and Sugar, 1921-1931

\begin{tabular}{|c|c|c|c|c|c|c|}
\hline \multirow{2}{*}{$\begin{array}{c}\text { Crop } \\
\text { year } \\
\text { begin- } \\
\text { ning }\end{array}$} & \multicolumn{2}{|c|}{ Wheat } & \multicolumn{2}{|c|}{ American Cotton } & \multicolumn{2}{|c|}{ Sugar } \\
\hline & $\begin{array}{l}\text { Supply, } \\
\text { million } \\
\text { bushels }\end{array}$ & $\begin{array}{c}\text { Disappear- } \\
\text { ance }\end{array}$ & $\begin{array}{c}\text { Supply, } \\
\text { million } \\
\text { bales }\end{array}$ & $\begin{array}{c}\text { Disappear- } \\
\text { ance }\end{array}$ & $\begin{array}{c}\text { Supply, } \\
\text { million } \\
\text { tons }\end{array}$ & $\begin{array}{l}\text { Disappear- } \\
\text { ance }\end{array}$ \\
\hline 1921 & 3485 & 3164 & 13.28 & 10.78 & 20.0 & 18.8 \\
\hline 1922 & 3477 & 3112 & 12.26 & 11.33 & 19.6 & 18.3 \\
\hline 1923 & 3867 & 3449 & 11.10 & 10.30 & 21.4 & 20.4 \\
\hline 1924 & 3499 & 3184 & 14.44 & 13.72 & 24.7 & 23.1 \\
\hline 1925 & 3654 & 3315 & 16.84 & 14.44 & 26.2 & 23.6 \\
\hline 1926 & 3759 & 3382 & 20.15 & 17.80 & 26.3 & 23.9 \\
\hline 1927 & 3977 & 3548 & 15.13 & 13.64 & 27.7 & 25.2 \\
\hline 1928 & 4340 & 3676 & 15.79 & 14.59 & 29.6 & 26.8 \\
\hline 1929 & 4095 & 3459 & 15.75 & 12.48 & 29.6 & 25.4 \\
\hline 1930 & 4435 & 3674 & 17.03 & 11.69 & & \\
\hline 1931 & 4465 & & & & & \\
\hline
\end{tabular}

Sources and method of calculation: Wheat: Wheat Studies of the Food Research Institute, July 1932, p. 422. From the supply, however, stocks in hands of European importers have been deducted, and to disappearance has been added the increase of those stocks. Am. Cotton: Bulletins on Cotton Production and Distribution of the Department of Commerce. Supplies are calculated from crops by adding stocks in public storage in the U. S. and stocks elsewhere in the U.S.; disappearances by subtracting from crops the increases in those stocks. 1921 and 1922 figures approximated from other sources. Sugar: "Studies in the Artificial Control of Raw Materials: Sugar"; by J. W. F. Rowe, London and Cambridge Economic Service.

To calculate $\tau$ it is necessary to make some assumption about $\bar{e}$. As already mentioned above, I have supposed $\bar{e}$ to be equal to the trend value of $v_{t}$. In the case of coffee a straight line has been taken as trend, drawn by hand, as it was of no importance to reach great accuracy. The slope of the trend for the pre-war period was chosen as 0.3 millions of bales, for the post-war period as 0.5. (See Table I.) To eliminate further long-run influences, $v_{t}$ as well as $s_{t}$ were calculated as percentages of the trend. From Fig. 1 it is seen that there exists a fairly good correlation between those two trend percentages, which enables us to evaluate $\tau$. There seem to be three distinctly different periods in any of which $\tau$ has a different value. By rough graphical calculation-a more accurate method seems to have no significance-I find:

for the period 1884-1898: about 2 years. 

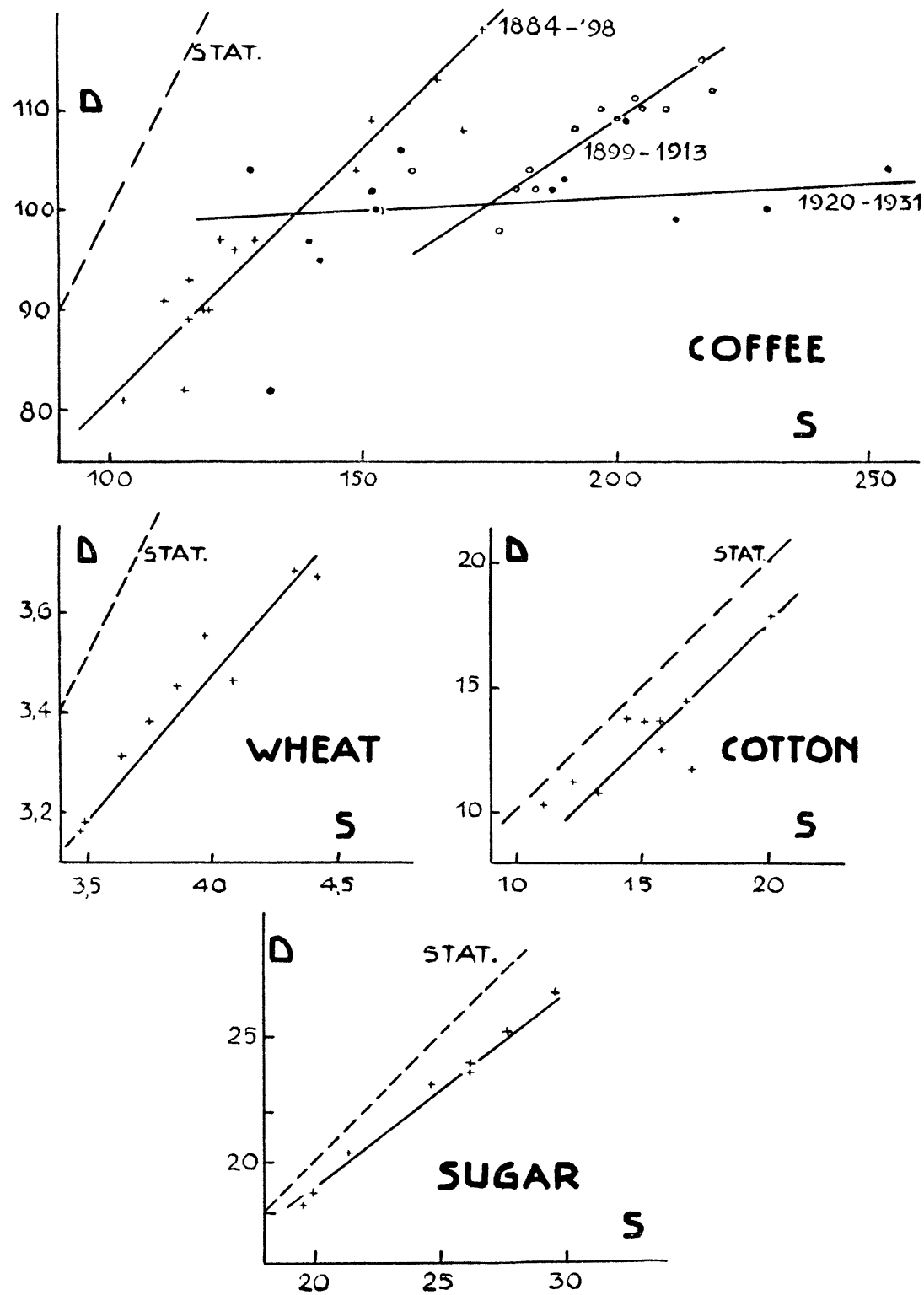

FigURE 1.-Relation between supply (=carry-over +crop) $S$ and disappearance $D$ during crop year. Coffee: $1884-1913$ and 1920-1931; wheat: 1921-1931, cotton: 1921-1930 and sugar: 1921-1929. For data see Tables I and II. The dotted line "Stat." indicates the relation demanded by static theory without taking account of "risk-stocks"; when those latter are included, the direction of the dotted lines still remains characteristic for the "static relation." 
for the period 1898-1914: about 3 to 4 years.

for the period 1921-1931: more than 10 years.

It is interesting to remark that Roth $^{3}$ indicates 1898 as the year in which the period of market equilibrium existing since 1887 was changed into a period of overproduction lasting until 1908. We can add that before the war a considerable decrease of stocks did not take place; and after the war, especially after 1925, overproduction grew still more serious. Our results may therefore be formulated: that in times of overproduction sellers in a still higher degree behave as if their horizon were widening.

For the cases of wheat, cotton, and sugar, only post-war figures were studied. It was not necessary here to eliminate trends, and it was supposed that $\bar{e}$ was simply a constant. Also, here there seems need for further detailed research. The provisional results found for $\tau$ were (cf. Table II and Fig. 1):

$$
\begin{aligned}
& \text { wheat: } 1.7 \text { years, } \\
& \text { cotton: about } 1 \text { year, } \\
& \text { sugar: } 1.3 \text { years. }
\end{aligned}
$$

RELATION BETWEEN DIVIDEND PAID ON CERTAIN STOCKS AND THEIR "WORTH"

By worth will be meant, in this paper, the product of a stock's price and the yield of state bonds. This product indicates the fixed interest to which the stock's yield is supposed to be equivalent. The relation between dividend really paid at a given moment $(D)$ and worth $(W)$ can only be established by static theory in the case the absolute certainty exists that the dividend will always remain the same; and then it is simply: $D=W$.

In the case of variable dividend, static theory cannot be applied. As a first approximation we can assume now, in accordance with the notions introduced above, that marginal buyers in the stock market have a definite horizon $\tau$; then a stock's worth will be determined by the dividend expectances during this horizon. This approximation will hold true especially for other than "highly speculative" buyers; for the latter will take account of, and probably take most account of, price expectance for the moment of re-selling. Supposing we have not to do with such speculative stocks, the question arises on what factors dividend expectancies will depend. Indications to the answer are given in the statistical material represented in Charts 2-6 and Tables III-VI.

${ }^{3} \mathrm{H}$. Roth, Die Übererzeugung in der Welthandelsware Kaffee im Zeitraum von 1790-1929, Jena, 1929. 


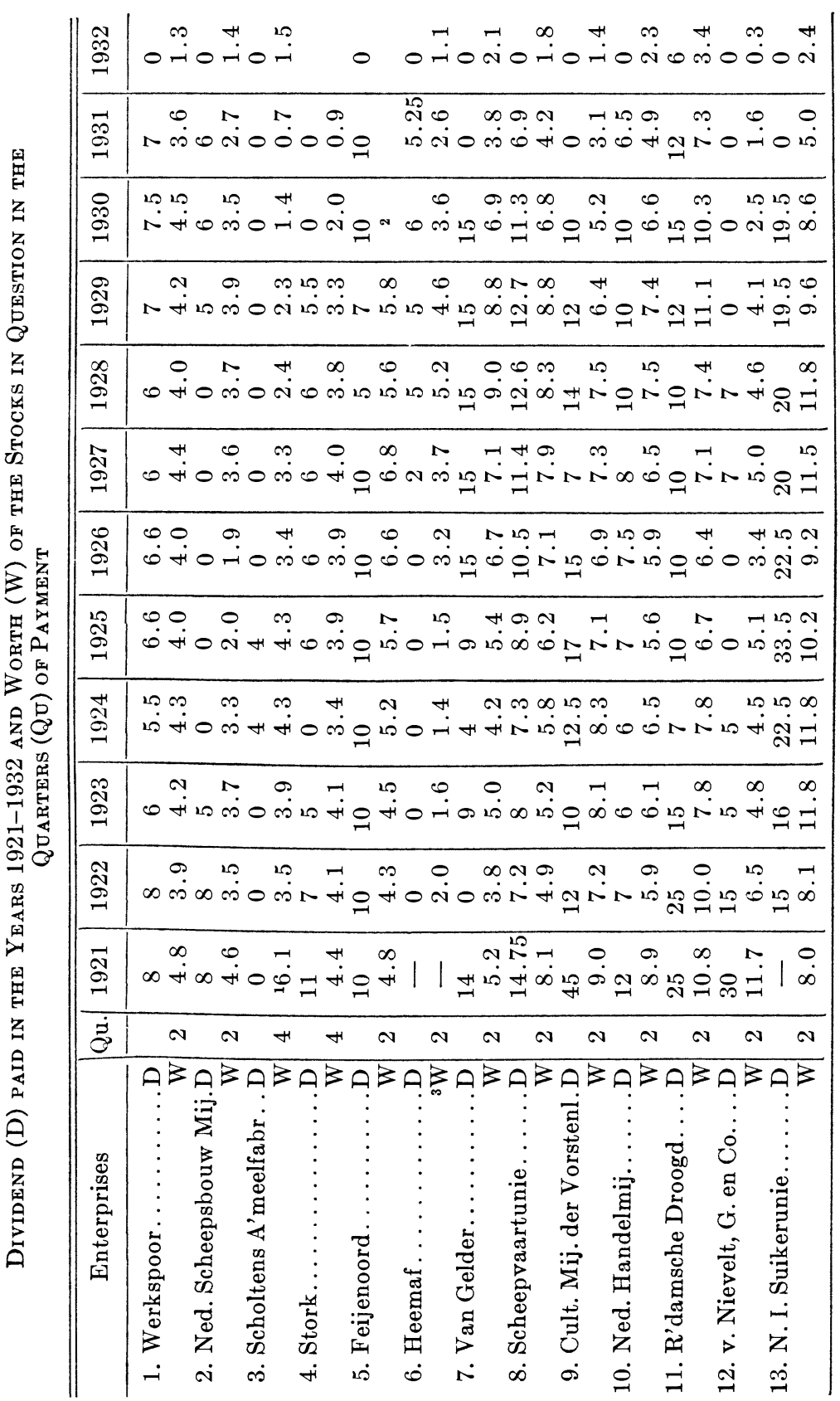


TABLE III, continued

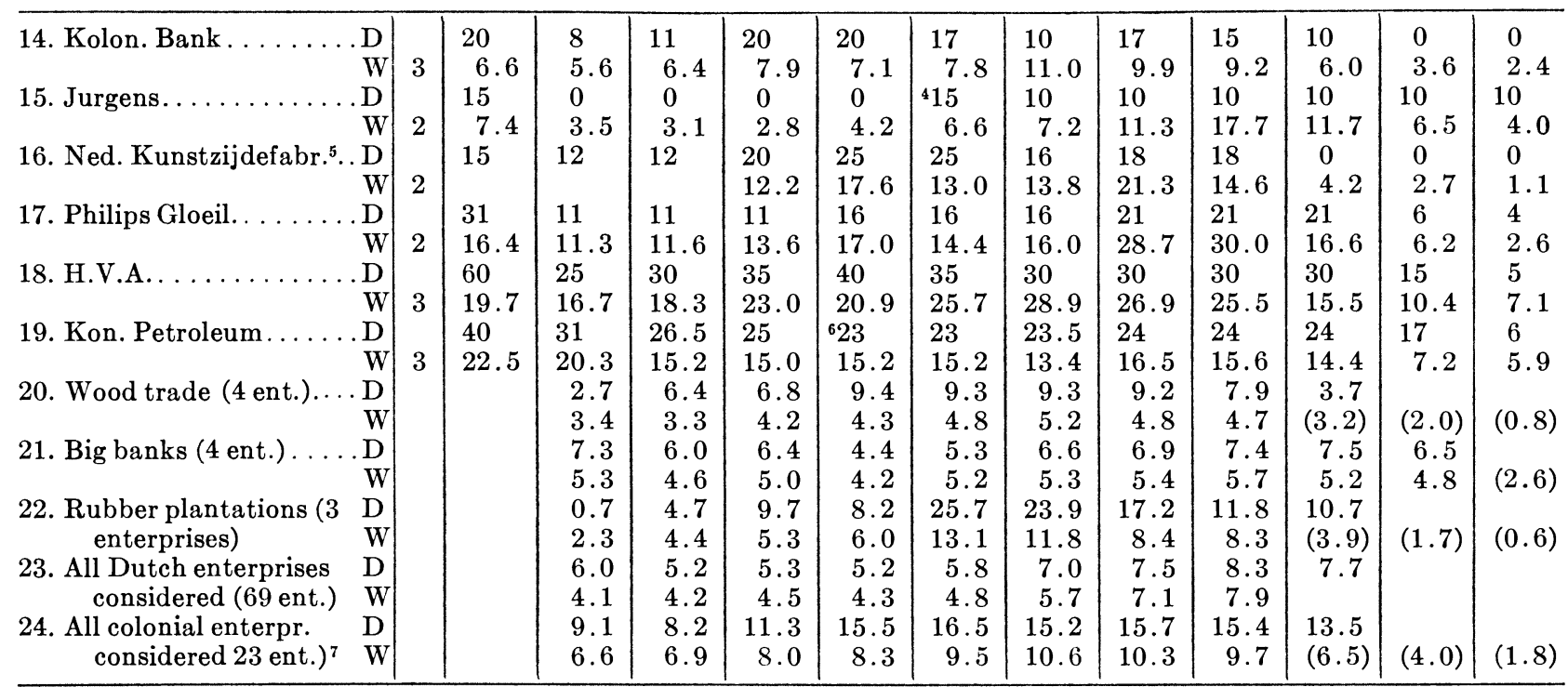

1 st quarter.

2 Amalgamation with Wilton's Shipbuilding Yard.

${ }^{3} \mathrm{Up}$ to $1926 \mathrm{~W}$ is given for the 4th quarter. Since, change of five year.

4 Combined dividend for 3 years.

5 Since 1929 Algemeene Kunstzijde Unie.

6 Old stocks only.

7 Without Petroleum Company. The "worth" of a stock is calculated by multiplying its price by the yield of 3 percent Dutch State Bonds. 


\begin{tabular}{|c|c|c|c|c|c|c|c|c|c|c|}
\hline & 兽 & 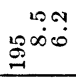 & 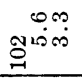 & 苟只 & $\theta_{7}^{+\infty}$ & مoois & న⿻ & 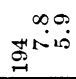 & $\mathscr{F}^{-10}$ & ガあ゙ず \\
\hline & 莽 & $\operatorname{s}^{-1}$ & הตึ่ & $\prod_{=1}^{\infty}$ & พัพ & ஜơ & मू० & 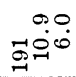 & $\hat{\infty}^{\cos } \hat{\alpha}$ & 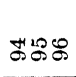 \\
\hline & ‡. & $\underbrace{\infty}_{\infty}-\infty i^{\infty}$ & H్ & $\stackrel{5}{s}^{N \infty}$ & 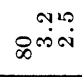 & $\mathbb{S}^{\infty 00}$ & शृ० & $\begin{array}{r}r+1 \\
-\infty \\
\infty\end{array}$ & $-_{0}^{\circ} \stackrel{9}{-1}$ & $88 \%$ \\
\hline & 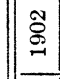 & 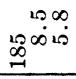 & 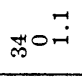 & $\stackrel{\overbrace{}}{\mathscr{c}}^{\mathrm{N} \infty}$ & ๓ஸं & $\mathbb{E}^{2000}$ & 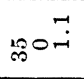 & $\begin{array}{l}\text { Ho } \\
000 \\
0\end{array}$ & 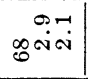 & 2085 \\
\hline 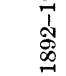 & $\vec{\Phi}$ & 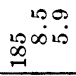 & [1 & $Z^{\infty}$ & ฌஸ் & $\underline{T}_{7}^{00}$ & นุN & $\stackrel{H \infty}{\infty} \stackrel{\infty}{\infty}^{\infty}$ & 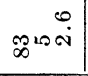 & のむ゙ず \\
\hline & \& & $g_{ت} \infty 0^{\circ}$ & 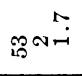 & $\mathbb{Z}^{\infty} \infty^{\infty}$ & क्ष & 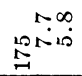 & fro & $\stackrel{\infty \infty}{\infty} \stackrel{\infty}{\infty}$ & $\infty_{\infty}^{L} \stackrel{\infty}{\sim}$ & దేరి \\
\hline 童 & 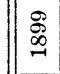 & $\operatorname{\sigma ్}^{\circ}$ & $* \stackrel{\leftrightarrow}{\mathrm{N} *}$ & $Z^{\infty}$ & 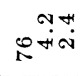 & พับ & भु & $\begin{array}{r}\sim \infty \\
\infty \infty \\
\stackrel{\infty}{\infty} \infty\end{array}$ & $\begin{array}{r}N 0 \\
\infty \\
N\end{array}$ & 8ஃஃே \\
\hline E & 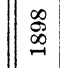 & $g_{9}^{100}$ & * ०* & $\stackrel{\infty}{\infty} \stackrel{0}{\infty}$ & 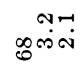 & gூo & & $\operatorname{son}_{0}^{20.1}$ & 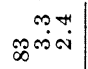 & 500 \\
\hline 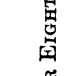 & 勇 & 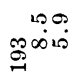 & ถึ่ & $\ddot{g}$ & 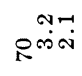 & 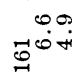 & ஜூ & $\stackrel{00}{\circ} \dot{\infty}$ & 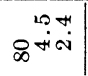 & ஃஃ8 \\
\hline$>\widehat{\varepsilon}$ & 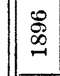 & 足 & מô. & $\underbrace{1000}$ & कळin & $R^{-7.7}$ & सम्冋 & 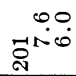 & $\begin{array}{r}\text { mo } \\
\hat{\sigma} \dot{\mathrm{N}}\end{array}$ & 우윰요 \\
\hline 国 & $\begin{array}{l}\stackrel{2}{\infty} \\
\stackrel{\infty}{-1}\end{array}$ & 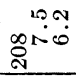 & $80^{\infty}-\overbrace{}^{-1}$ & $\check{g}^{\circ \circ}$ & $\mathscr{\infty}$ & 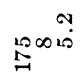 & تరం & क्षें & Nơ & 후워음 \\
\hline & $\underset{\infty}{\stackrel{0}{\infty}}$ & 苟 & 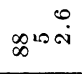 & $8^{100}$ & $\begin{array}{r}\ddot{\infty}+\infty \\
\sigma\end{array}$ & ${ }_{5}^{\infty}$ & 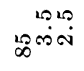 & 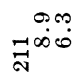 & J̆س & 5엉오 \\
\hline 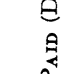 & $\stackrel{\mathscr{D}}{\stackrel{\infty}{-}}$ & : & $\underset{\infty}{\mathscr{\infty}} \stackrel{\dot{N}}{ }$ & S० & 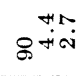 & تص心 & ஜடா & 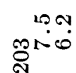 & go: & $\stackrel{\infty}{\infty}: \infty$ \\
\hline$\sum$ & 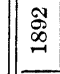 & $\sin _{-10 \infty}^{n+\infty}$ & $\mathscr{\infty}_{\infty}^{1000}$ & 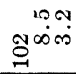 & ${ }_{\infty}^{+\infty i \infty}$ & $\mathbb{E}^{\infty}$ & Dơ & $\begin{array}{r}\infty 0 . \\
\dot{\infty} \infty \dot{0}^{\circ}\end{array}$ & $\begin{array}{r}0 \\
\sigma\end{array}$ & ガல゙வ \\
\hline & & $\triangle A B$ & $\triangle A B$ & 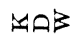 & GAB & जaß & $\triangle A B$ & $\Leftrightarrow A B$ & $\triangle A B$ & 1 \\
\hline 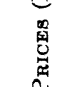 & 急 & $\Xi$ & : & 3 & $\stackrel{\vec{\Phi}}{\sum_{i}^{2}}$ & $\vec{g}$ & $\overrightarrow{3}$ & 当 & $\exists$ & 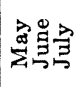 \\
\hline 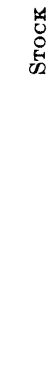 & 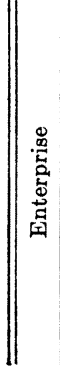 & 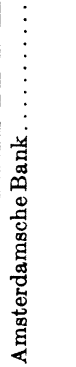 & 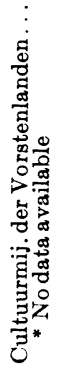 & 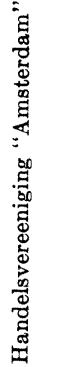 & 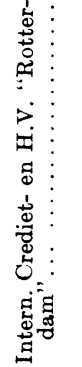 & 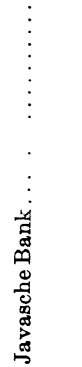 & 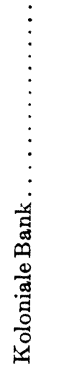 & 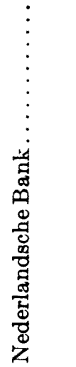 & 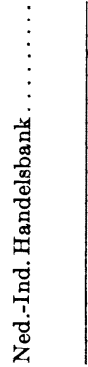 & 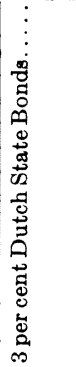 \\
\hline
\end{tabular}




\begin{tabular}{|c|c|c|c|c|c|c|c|c|c|}
\hline 家 & 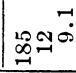 & ูำ่อ๋ & จำล & 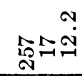 & ㅇำ & $\stackrel{ }{N}^{n} \stackrel{0}{0}$ & $\underset{\infty}{\infty} \dot{\Omega}^{\circ}$ & $\underset{\infty}{\infty}=\stackrel{\sim}{\sim}$ & 808 \\
\hline$\stackrel{\infty}{\sigma}$ & & $\exists 0^{5}$ & 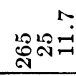 & $\vec{a}=\overrightarrow{0}$ & 소ㅇㅛㅛ & $\sigma^{20}+\operatorname{ti}^{\circ}$ & 足 & 20$)^{\infty} \underbrace{\infty}$ & $\otimes \otimes^{\infty}$ \\
\hline 를 & 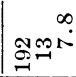 & สู่న & สูำ & $\underset{\mathcal{N}}{*}=\overrightarrow{\sigma^{\circ}}$ & 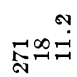 & 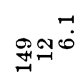 & No & $\operatorname{a্d}^{\infty} \infty^{\infty}$ & ペ梠 \\
\hline$\stackrel{0}{\sigma}$ & $\underset{D}{\infty}=\stackrel{0}{2}$ & $\sin ^{\infty} \sin ^{\infty}$ & ஜ্ׁల & $\mathscr{N}_{0}^{\infty}+\mathbb{1}$ & ت్న & 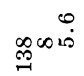 & ผู & 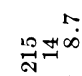 & ペボ \\
\hline$\frac{2}{9}$ & goi & 禹 & న్ని & 촌 & 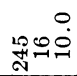 & $\mathbb{S}^{0}$ & 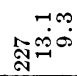 & $\underset{\sim}{\infty} \stackrel{+}{\stackrel{4}{r}}$ & אNי \\
\hline 节 & ํำ & فำ & 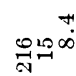 & مْ & भर० & ن. & 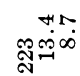 & 도요 & $\underset{\infty}{\infty} \stackrel{\infty}{\wedge}$ \\
\hline$\stackrel{m}{\stackrel{9}{9}}$ & $\underset{\infty}{\infty}=\stackrel{+}{n}$ & ஜ్రిం & $\overbrace{-\infty}^{\infty}$ & 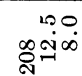 & 웜혀 & 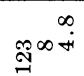 & พื⿻ & 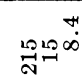 & 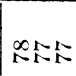 \\
\hline$\underset{\Xi}{\stackrel{乛}{\Xi}}$ & go. & $120^{\circ}$ & \&: & ถูำ & 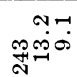 & 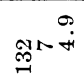 & N & $\begin{array}{l}100 \\
\infty \infty^{\infty} \infty \\
\infty \rightarrow \infty\end{array}$ & $-\infty \otimes \infty$ \\
\hline$\vec{\sigma}$ & 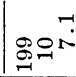 & 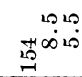 & ลู่อ & 부요 & $\vec{\infty} \underset{\infty}{\infty}$ & $\hat{\mathbb{N}}^{N}+\frac{0}{+}$ & $\overbrace{i}=0$ & :్ల & $10+\infty \infty$ \\
\hline 음 & $\overbrace{}^{\circ} 0^{\circ}$ & 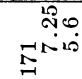 & $a^{\infty}{ }^{n}$ & $\begin{array}{l}\text { Ho } \\
0.0\end{array}$ & مै? & $\vec{m}^{N} \stackrel{H}{H}^{n}$ & $\sigma^{\infty}$ & 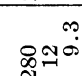 & 6ప8 \\
\hline \& & $\sin ^{\infty}$ & "艹 & มู่า & ${ }_{10}^{+00}$ & 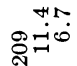 & & $\begin{array}{r}\infty 0 \\
\mathbb{S}_{\infty}^{\infty} \infty 0^{\circ}\end{array}$ & 꾹ำ & 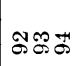 \\
\hline$\stackrel{\infty}{\circ}$ & स्रक & $\overbrace{7}^{1000}$ & ". & 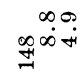 & $8 \forall 0^{\infty}$ & $\overrightarrow{0}^{\circ}$ & & $\mathbb{J}^{\infty}{ }^{\infty}+\mathfrak{r}$ & ৪৪৪ \\
\hline 今 & $\sigma_{\theta}=\stackrel{+}{=}$ & 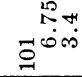 & : & $\underset{J}{+\infty} \stackrel{\infty}{\infty}$ & $\underset{\infty}{\infty} \underset{\sim}{2}=\stackrel{\sim}{\circ}$ & $\infty_{\infty}^{\infty} \stackrel{\infty}{\circ}$ & $\stackrel{0}{2} \stackrel{0}{=}$ & 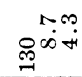 & $\infty \infty 8$ \\
\hline 导 & sing & Sin' & مْ & 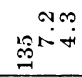 & 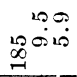 & Nละ & 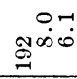 & สำ & むేరు \\
\hline & $\angle A$ & $\triangle A B$ & $\forall A$ & $\because A=$ & $\angle A B$ & $\triangle A B$ & $\triangle A B$ & $M A B$ & $10 x+4$ \\
\hline 韋 & 㿣 & 号 & 弯 & $\overrightarrow{\Sigma^{\circ}}$ & $\overrightarrow{3}$ & $\stackrel{3}{3}$ & $\stackrel{\partial}{\exists}$ & 3 & 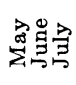 \\
\hline 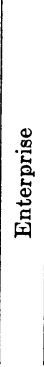 & 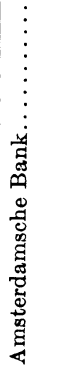 & 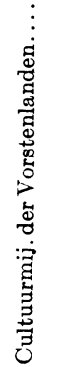 & 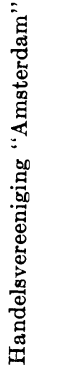 & 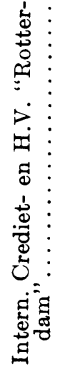 & 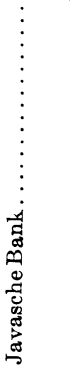 & 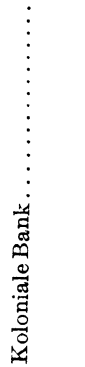 & 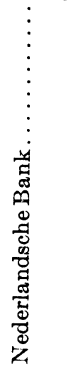 & 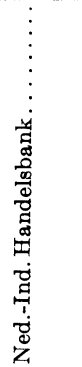 & 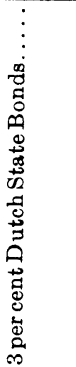 \\
\hline
\end{tabular}


TABLE V

Stock Prices and Dividends Paid, 1921-1932, for Three Java Rubber Plantations

\begin{tabular}{|c|c|c|c|c|c|c|c|c|c|c|c|c|c|c|}
\hline \multirow{2}{*}{ Enterprise } & \multirow{2}{*}{ Year } & \multicolumn{12}{|c|}{ Stock prices } & \multirow{2}{*}{$\mid \begin{array}{l}\text { Divi- } \\
\text { dends }\end{array}$} \\
\hline & & Jan. & Feb. & March & Apr. & May & June & July & Aug. & Sept. & Oct. & Nov. & Dec. & \\
\hline $\begin{array}{l}\text { 1. Amsterdam } \\
\text { Rubbercul- } \\
\text { tuur Maat- } \\
\text { schappij }\end{array}$ & $\begin{array}{l}1921 \\
1922 \\
1923 \\
1924 \\
1925 \\
1926 \\
1927 \\
1928 \\
1929 \\
1930 \\
1931 \\
1932\end{array}$ & $\begin{array}{r}141 \\
101 \\
138 \\
153 \\
165 \\
356 \\
331 \\
319 \\
262 \\
160 \\
103 \\
47\end{array}$ & $\begin{array}{r}134 \\
93 \\
149 \\
163 \\
167 \\
356 \\
332 \\
295 \\
282 \\
175 \\
109 \\
46\end{array}$ & $\begin{array}{r}116 \\
85 \\
149 \\
148 \\
189 \\
344 \\
351 \\
264 \\
275 \\
148 \\
111 \\
41\end{array}$ & $\begin{array}{r}95 \\
87 \\
165 \\
143 \\
211 \\
331 \\
333 \\
233 \\
260 \\
152 \\
88 \\
30.6\end{array}$ & $\begin{array}{r}105 \\
78 \\
155 \\
132 \\
225 \\
332 \\
342 \\
239 \\
264 \\
141 \\
83 \\
30\end{array}$ & $\begin{array}{c}86 \\
61 \\
149 \\
117 \\
229 \\
313 \\
307 \\
230 \\
256 \\
121 \\
73 \\
29.9\end{array}$ & $\begin{array}{r}96 \\
52 \\
145 \\
133 \\
284 \\
323 \\
288 \\
230 \\
261 \\
123 \\
86 \\
42\end{array}$ & $\begin{array}{r}97 \\
66 \\
152 \\
147 \\
285 \\
318 \\
304 \\
234 \\
266 \\
105 \\
68 \\
62\end{array}$ & $\begin{array}{r}91 \\
75 \\
155 \\
151 \\
318 \\
334 \\
298 \\
228 \\
266 \\
89 \\
50 \\
68\end{array}$ & $\begin{array}{r}94 \\
98 \\
144 \\
159 \\
368 \\
333 \\
293 \\
231 \\
254 \\
99 \\
54 \\
62\end{array}$ & $\begin{array}{r}104 \\
110 \\
147 \\
158 \\
397 \\
324 \\
311 \\
236 \\
200 \\
110 \\
54\end{array}$ & $\begin{array}{r}106 \\
116 \\
145 \\
162 \\
386 \\
316 \\
323 \\
232 \\
167 \\
104 \\
44\end{array}$ & $\begin{array}{r}0 \\
0 \\
8 \\
12 \\
15 \\
25 \\
25 \\
18 \\
15 \\
15 \\
0 \\
0\end{array}$ \\
\hline $\begin{array}{l}\text { 2. Serbadjadi- } \\
\text { Sumatra- } \\
\text { Rubber-cul- } \\
\text { tuurmij }\end{array}$ & $\begin{array}{l}1921 \\
1922 \\
1923 \\
1924 \\
1925 \\
1926 \\
1927 \\
1928 \\
1929 \\
1930 \\
1931 \\
1932\end{array}$ & $\begin{array}{c}230 \\
221 \\
280 \\
227 \\
214 \\
382 \\
337 \\
328 \\
214 \\
118 \\
62 \\
19.3\end{array}$ & $\begin{array}{l}229 \\
213 \\
285 \\
234 \\
214 \\
377 \\
335 \\
303 \\
238 \\
127 \\
68 \\
19.5\end{array}$ & $\begin{array}{c}197 \\
208 \\
293 \\
211 \\
220 \\
365 \\
352 \\
256 \\
235 \\
113 \\
71 \\
15.0\end{array}$ & $\begin{array}{c}158 \\
211 \\
323 \\
202 \\
223 \\
348 \\
344 \\
205 \\
203 \\
120 \\
48 \\
7.1\end{array}$ & $\begin{array}{c}175 \\
202 \\
288 \\
188 \\
240 \\
343 \\
339 \\
204 \\
200 \\
105 \\
36 \\
7.8\end{array}$ & $\begin{array}{c}139 \\
193 \\
282 \\
177 \\
238 \\
313 \\
306 \\
201 \\
187 \\
85 \\
37 \\
7.0\end{array}$ & $\begin{array}{c}150 \\
170 \\
284 \\
175 \\
278 \\
325 \\
291 \\
178 \\
203 \\
84 \\
48 \\
12.5\end{array}$ & $\begin{array}{c}176 \\
184 \\
297 \\
194 \\
267 \\
322 \\
310 \\
185 \\
205 \\
70 \\
34 \\
24.9\end{array}$ & $\begin{array}{l}169 \\
197 \\
299 \\
193 \\
300 \\
339 \\
299 \\
172 \\
206 \\
58 \\
20.4 \\
30\end{array}$ & $\begin{array}{c}177 \\
232 \\
286 \\
194 \\
363 \\
337 \\
288 \\
175 \\
197 \\
59 \\
25.5 \\
22.5\end{array}$ & $\begin{array}{c}197 \\
240 \\
290 \\
193 \\
426 \\
328 \\
306 \\
188 \\
143 \\
64 \\
23.8\end{array}$ & $\begin{array}{c}227 \\
267 \\
262 \\
203 \\
410 \\
321 \\
329 \\
181 \\
120 \\
62 \\
19.1\end{array}$ & $\begin{array}{r}0 \\
17 \\
20 \\
18 \\
17 \\
30 \\
25 \\
20 \\
8 \\
0 \\
0 \\
0\end{array}$ \\
\hline $\begin{array}{l}\text { 3. Deli-Batavia } \\
\text { Rubbermij }\end{array}$ & $\begin{array}{l}1921 \\
1922 \\
1923 \\
1924 \\
1925 \\
1926 \\
1927 \\
1928 \\
1929 \\
1930 \\
1931 \\
1932 \\
\end{array}$ & $\begin{array}{r}111 \\
71 \\
82 \\
82 \\
108 \\
257 \\
265 \\
252 \\
179 \\
95 \\
54 \\
19.7\end{array}$ & $\begin{array}{r}106 \\
64 \\
81 \\
82 \\
110 \\
255 \\
269 \\
235 \\
196 \\
110 \\
60 \\
19.3\end{array}$ & $\begin{array}{r}90 \\
58 \\
82 \\
83 \\
118 \\
241 \\
278 \\
199 \\
191 \\
107 \\
63 \\
14.8\end{array}$ & $\begin{array}{r}73 \\
56 \\
90 \\
79 \\
122 \\
235 \\
271 \\
165 \\
173 \\
107 \\
44 \\
7.6\end{array}$ & $\begin{array}{c}85 \\
47 \\
84 \\
72 \\
140 \\
241 \\
281 \\
168 \\
173 \\
93 \\
41 \\
8.8\end{array}$ & $\begin{array}{c}67 \\
37 \\
75 \\
68 \\
147 \\
228 \\
254 \\
161 \\
164 \\
77 \\
29 \\
8.5\end{array}$ & $\begin{array}{c}71 \\
29 \\
76 \\
75 \\
180 \\
248 \\
234 \\
154 \\
172 \\
78 \\
42 \\
15.5\end{array}$ & $\begin{array}{r}77 \\
37 \\
79 \\
83 \\
179 \\
244 \\
248 \\
164 \\
175 \\
62 \\
31 \\
27.5\end{array}$ & $\begin{array}{r}66 \\
40 \\
78 \\
87 \\
211 \\
267 \\
242 \\
152 \\
174 \\
46 \\
19 \\
33\end{array}$ & $\begin{array}{c}65 \\
59 \\
73 \\
88 \\
241 \\
269 \\
243 \\
155 \\
168 \\
50 \\
21.2 \\
27.3\end{array}$ & $\begin{array}{r}71 \\
65 \\
76 \\
87 \\
281 \\
259 \\
252 \\
163 \\
127 \\
60 \\
21.5\end{array}$ & $\begin{array}{c}74 \\
72 \\
78 \\
91 \\
273 \\
250 \\
254 \\
160 \\
103 \\
54 \\
18.4\end{array}$ & $\begin{array}{r}0 \\
0 \\
0 \\
0 \\
0 \\
15 \\
20 \\
10 \\
10 \\
0 \\
0 \\
0\end{array}$ \\
\hline
\end{tabular}




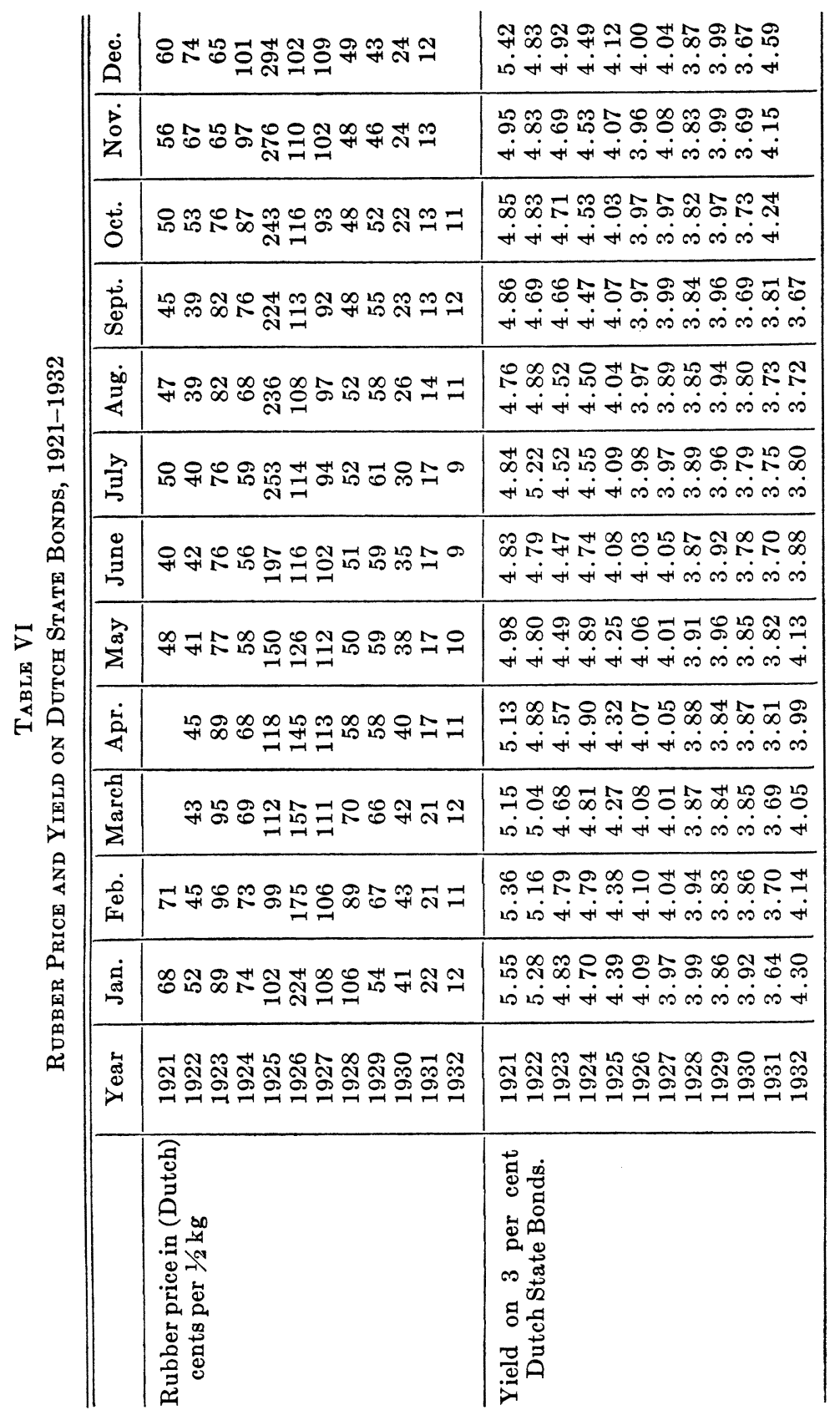




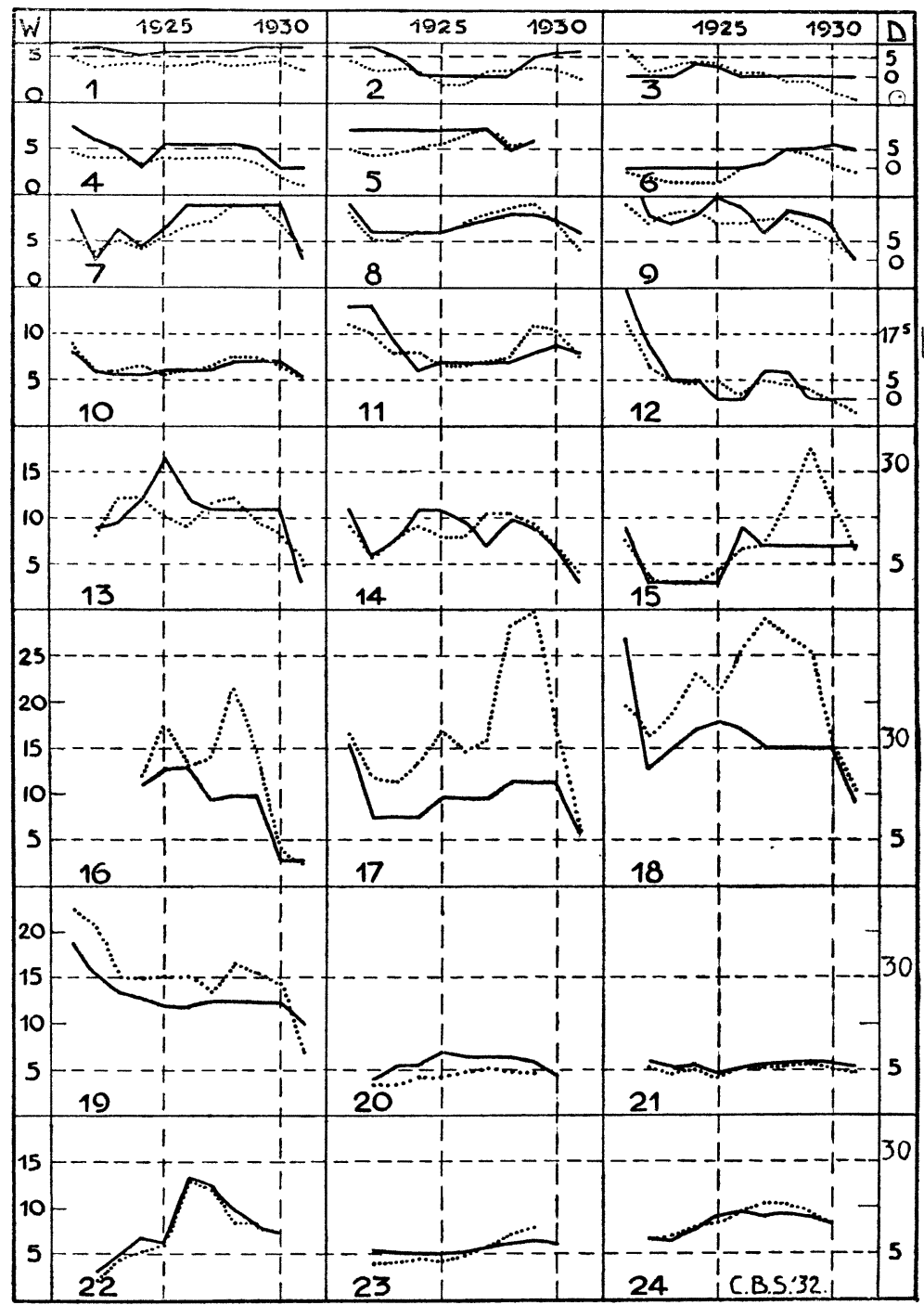

Figure 2. ....... Dividend $(D)$ paid, and - Worth $(W)$ in the quarter of dividend payment, for 19 Dutch enterprises and 5 groups of enterprises, 1921-1932.

The scale of this chart has been chosen in such a way that the lines cover each other when the relation

$$
W=3+0.4 D \text {. }
$$

is satisfied. This relation represents the relation found for the totality of the material, by rough estimate.

The explanation of the numbers and the figures for $D$ and $W$ are given in Table I. 
Figs. 2 and 3 and Table III relate to a number of Dutch stocks, selected out of several branches of industry, for the period 1921-1931. For each stock considered, $D$ and $W$ have been calculated; figures relate to the month in the middle of the quarter in which dividend was paid. ${ }^{4}$ The calculations were made for 19 leading companies separately and, in addition, for 5 groups of enterprises, as indicated in Table III. The results are represented graphically in two ways. Fig. 2 compares, for each case, $D$ and $W$. The scales for $D$ and $W$ are taken different, such that the curves for $D$ and $W$ are identical when the relation

$$
W=3+0.4 D
$$

is satisfied. This was, roughly estimated, the relation found for the majority of cases to hold fairly well. So Fig. 2 shows the degree of ac-

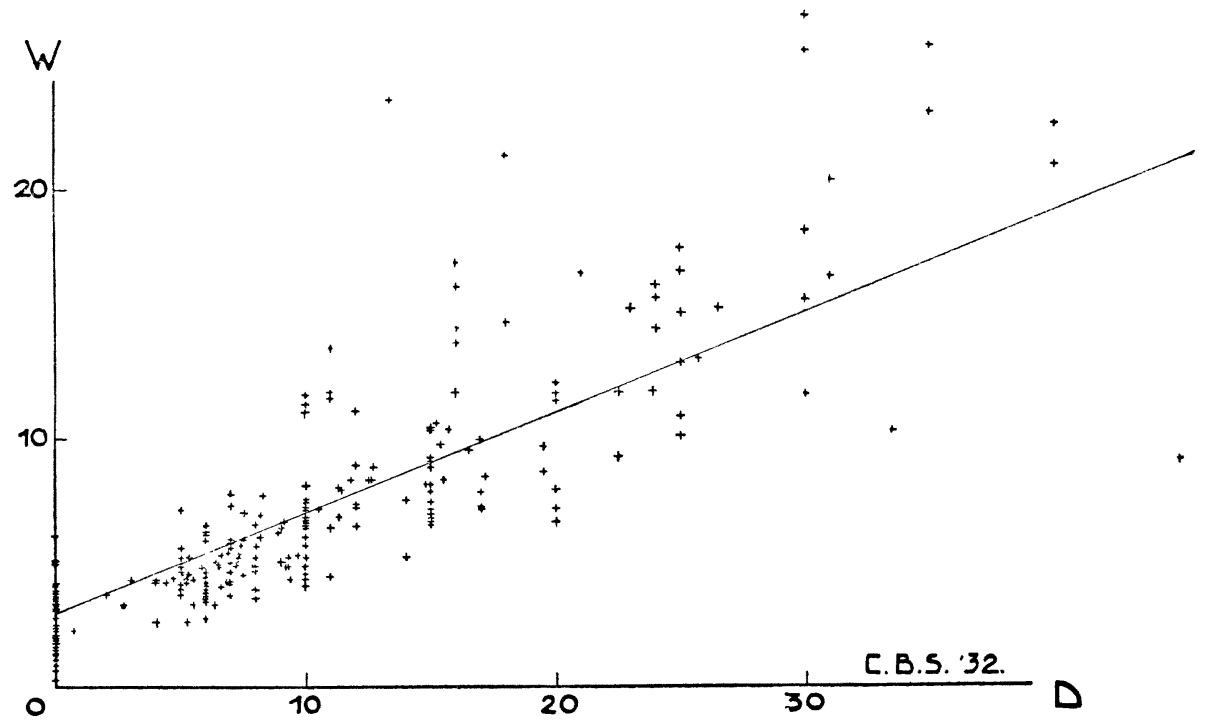

FigURe 3.- $D$ and $W$ for the enterprises and groups considered (1921-1932). Straight line:

$$
W=3+0.4 D
$$

cordance for the individual stocks. It is interesting that the deviations from relation (1) are largest for the really speculative stocks Nos. $15-19$.

In Fig. 3, corresponding values of $D$ and $W$, as given in Table III

${ }^{4}$ This method of calculation was chosen for reasons of simplification. A number of tests indicated that no great differences occur when other possible methods are followed. 
and Fig. 2, have been plotted against each other. The same has been done for 8 stocks (banking and colonial agriculture only) for which data were easily to be found, in the period 1892-1919. The result is not very different. Owing to rough graphical calculation the relation between $D$ and $W$ for these stocks is about (cf. Fig. 4):

$$
W=1.5+0.47 D
$$

The significance of the result, in connection with the fairly good correlation between the two members of the equations given, may be formulated as follows: The chief determinant factor to $W$, i.e., to dividend expectancies, is the last dividend paid. ${ }^{5}$ The movements of $D$, however, are reflected only about half as intensively. This shows that reactions are always expected in about such a way that the total picture of the future corresponds to a dividend of about "half as abnormal" as the last dividend paid. As "normal" dividend $D_{0}$ occurs, then, the dividend for which $W=D$, i.e., 5 per cent for the decade after the war, and about 3 per cent for pre-war years.

The most interesting feature in our result is illustrated by Fig. 7 . To fix the ideas, $\tau$ is indicated by the distance shown at the bottom. $D$ may indicate the real course of dividend through a business cycle. As we just have seen that for the period $\tau$ there exists an average dividend expectance equivalent to a "normal" dividend $D_{0}$ plus about half the excess of $D$ over $D_{0}$, we can, also, to fix the ideas, suppose that the expected course of $D$ at each moment is indicated by the dotted lines. The essential feature of those lines is that something like the surface between each such line and the $D_{0}$-axis should be proportional to the height of $D$ over $D_{0}$. Although a conclusion might be premature, this diagram suggests the absence of any "forecasting quality" of stock prices.

\section{III}

\section{SHORTER MOVEMENTS OF STOCK PRICES}

The above-considered relation between $D$ and $W$ only determines the annual movement of $W$, and therefore of stock prices. For shorter movements, other determining factors exist, which is illustrated by Figs. 5 and 6 for rubber plantation stocks. Fig. 5 again shows the agreement between $D$ and (twelve month averages of) $W$; Fig. 6 illustrates the correlation between the "shorter movements" of $W$ (viz. deviations from 12 months' averages) and those of rubber prices $P$. (Numbers 1, 2 and 3 relate to three different enterprises.)

${ }^{5}$ Cf. also Wochenbericht des Instituts für Konjunkturforschung, 15 Nov. 1932, p. 133, giving similar results of an investigation by Dr. O. Donner. 
As to the correlation shown in Fig. 6, the following remarks may precede our further considerations: The best agreement exists between $W_{i}$ and percentage values of $P$ (i.e., $P$ in per cent of 12 months' moving

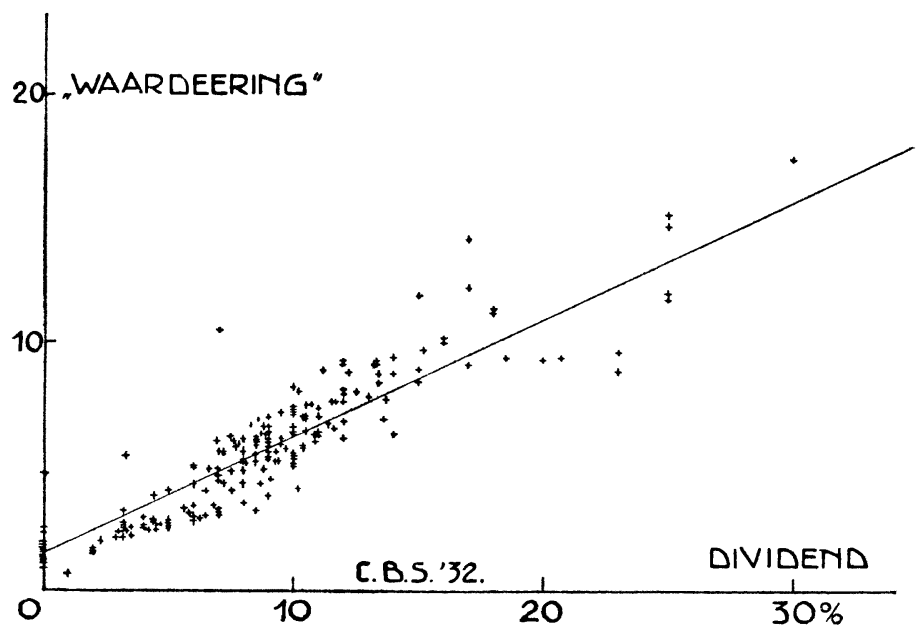

FIgURE 4. $-D$ and $W$, for the enterprises considered, (1892-1919). Straight line:

$W=1.5+0.47 D$.

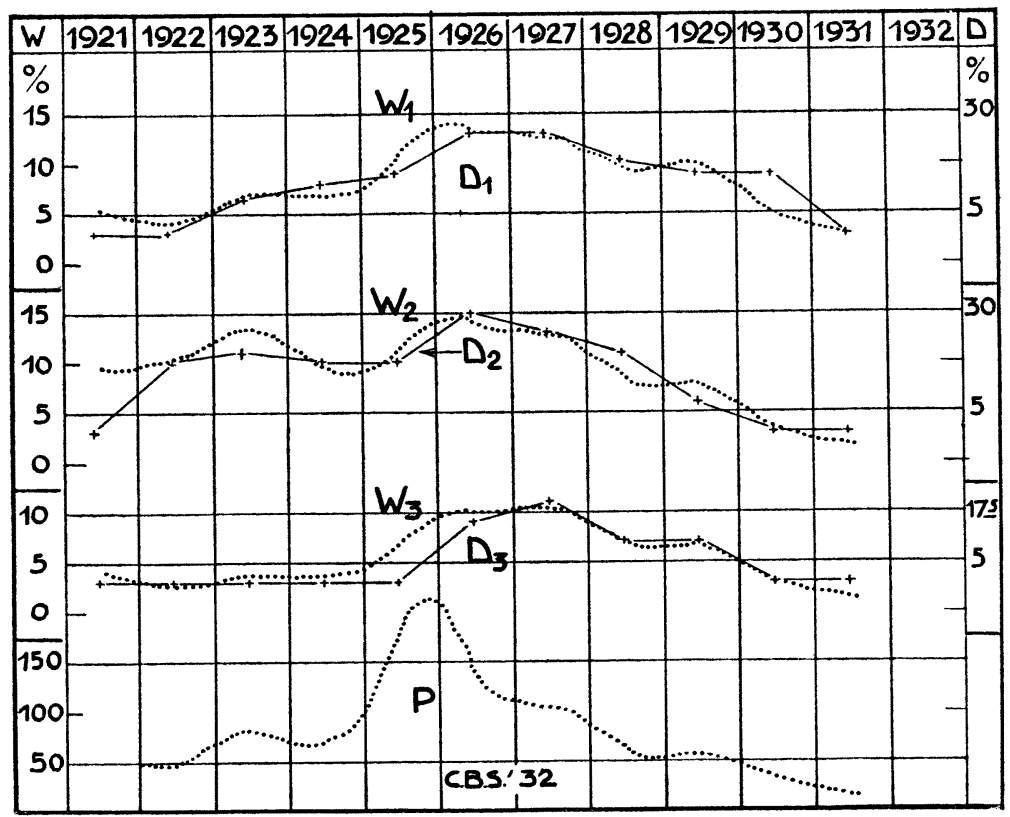

Figure 5.-W and $D$ for three Java rubber plantations, 1921-1932. W's: 12 months' moving averages. The indexes relate to the three enterprises mentioned in Table III. Scale similar to that of Fig. 1. 
averages, heavy line); logically one should expect $W$ to correlate with the absolute price movements (as equal absolute price increments cause equal dividend increments), indicated by the dotted line. This correlation is, however, far poorer; and it remains still a little poorer when a multiple correlation between $W$ on one side and $P$ and $K$ on

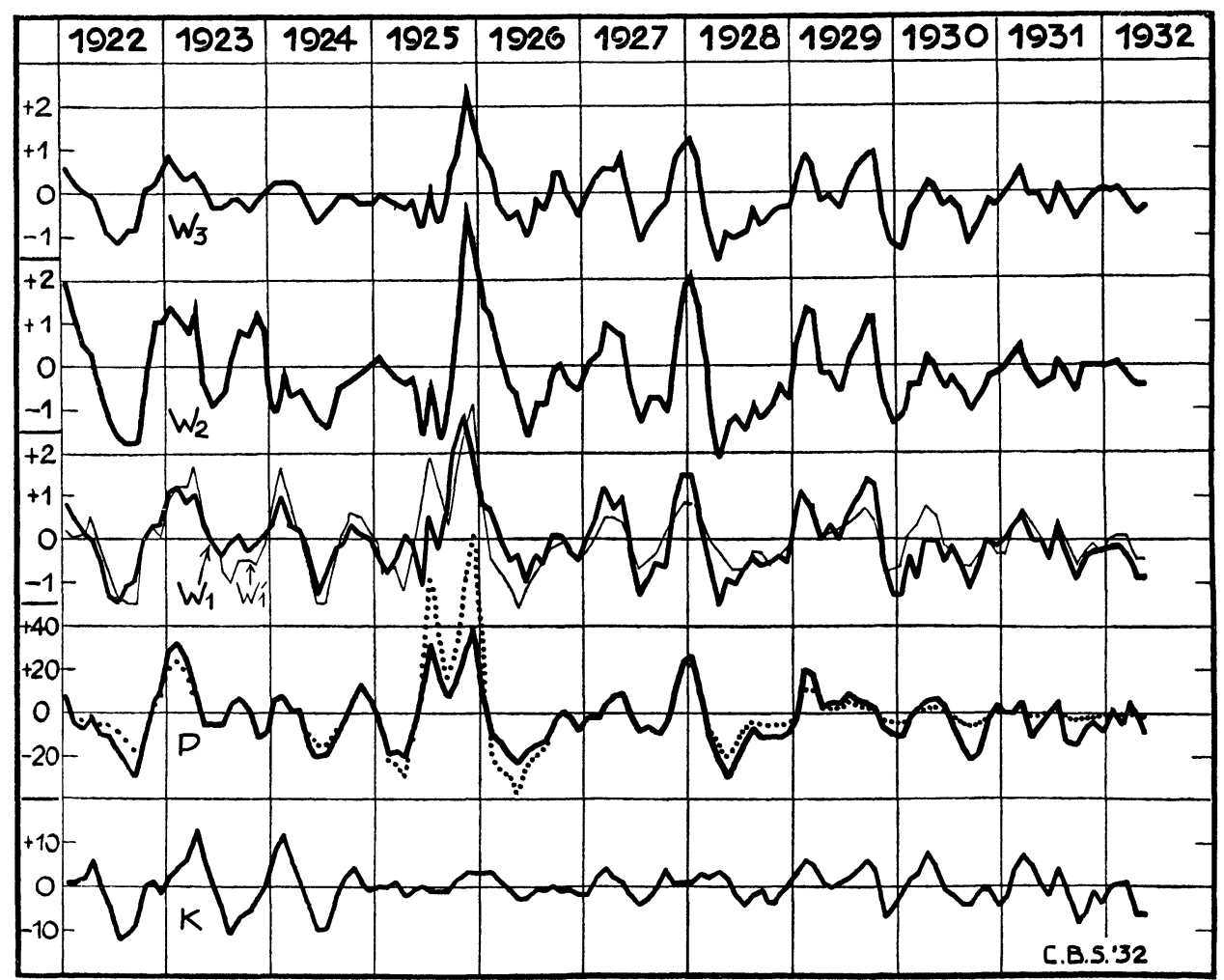

FIGURE 6.-Short fluctuations of worth of rubber stocks, $W$, as compared with short fluctuations in rubber price, $P$, and in general index of stock prices, $K(1921-25=100)$. The indexes relate to the enterprises mentioned in Table III. All series: deviation from 12 months' moving averages. Heavy line $P$ : percentage deviations; dotted line $P$ : absolute deviations, in cts per $\frac{1}{2} \mathrm{~kg} . W_{1}^{\prime}$, calculated from formula:

$$
W_{1}{ }^{\prime}=0.035 P+0.097 K
$$

in which $P$ represents absolute price deviations, giving maximum correlation with $W_{1}$.

the other side is considered $-K$ indicating general stock price index (deviations from 12 months' averages). This is shown by the line $W_{1}{ }^{\prime}$, giving the "best combination" of $K$ and (dotted) $P$. As far as I can see a simple explanation of this fact is not easy. 
We may consider the matter also from another angle. Supposing the correlation between the absolute price deviations and $W$ is satisfactory, what is then to be deduced, with the help of our notions "horizon" and "expectancy," from the regression coefficient?

From that coefficient we deduce that, for the short movements, 1 cent of price difference (per $\frac{1}{2} \mathrm{~kg}$ ) corresponds to a difference in 0.035 per cent of $W_{1}$, i.e., of dividend expectance during the period of $\tau$. Now from other sources that are known to every investor, viz. the profit and loss account, ${ }^{6} \mathrm{I}$ deduce that a price movement of 1 cent causes a dividend movement (during the period 1921-1931, with large increases and large decreases) of 0.3 per cent. If the investor expected the deviation of price from normal to apply for his whole $\tau$, then he had to expect, for this $\tau$, a dividend of 0.3 per cent multiplied by the

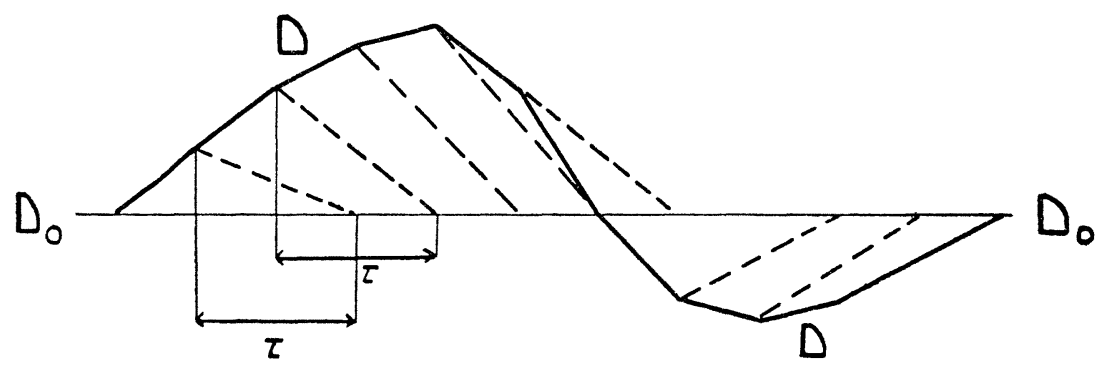

FIGURe 7.-Character of Dividend expectances. $D$ : actual course of dividends. Dotted lines: expectances at each moment; $r$ : length of "horizon," chosen arbitrarily. The essence of the scheme is that at each moment expectances gravitate to $D_{0}$, a sort of "normal" dividend.

price deviation in cents. As he only seems to expect 0.035 per cent, our conclusion is that he expects price to move back again within the period $\tau$, such that "on the average" only a deviation of about 0.1 of the deviation observed will exist.

So, although it was not possible in the second and third problem presented to evaluate the horizon, it seems, nevertheless, possible to learn something about the expectancy.

University of Amsterdam, Holland

- See e.g. Van Oss' Effectenboek 1932 I, 1084-1088. 